\title{
Placental histology findings in relation to pre-eclampsia: implications for interpretation of retrospective studies
}

\author{
Prof. Neil Sebire \\ Department of Histopathology, Great Ormond Street Hospital for Children NHS Trust, \\ London, UK \\ neil.sebire@gosh.nhs.uk
}

In this issue Khalil et al. report the findings of a systematic review of studies reporting placental histological features in relation to pre-eclampsia (PET). ${ }^{1}$ The main findings of this study were first, that whilst certain histological patterns were more common in PET than controls, similar features were also noted in unaffected cases, and secondly, that the results were related to whether the histological features in the studies were assessed blindly, with significantly greater apparent differences between groups reported in unblinded studies. These results demonstrate the impact of bias, which may be unconscious, on study outcome, with important implications for appropriate interpretation of not only placental pathology studies, but all such studies with a potentially subjective element of interpretation.

The scientific investigation of errors in how we think and make decisions is now well established, ${ }^{2}$ this area resulting in the Nobel prize in economics in $2002,{ }^{3}$ although there is relatively little published specifically in relation to medicine in relation to thinking and interpretation. Potential pitfalls described in general include subconscious bias without blinding, selection bias, confirmation bias, hindsight bias and many more. ${ }^{4}$

Specifically, there are several areas of potential methodological difficulty with retrospective studies of placental findings. First, many such studies are based on review of a subgroup of cases which were submitted as part of clinical care, usually to a specialist centre, which results in marked preselection of pregnancies, increasing the proportion of affected cases in the overall group. Whilst this in itself may not necessarily affect the odds ratio for detecting associations between histological features and outcome, it has a significant effect on the positive predictive value of the test when used in clinical practice and hence the overall strength of association. True characteristics of such associations can only be determined from studies in which large, unselected populations are included rather than highly preselected groups based on pregnancy complications, which by definition enhance for the outcome in question. ${ }^{5}$

This article has been accepted for publication and undergone full peer review but has not been through the copyediting, typesetting, pagination and proofreading process, which may lead to differences between this version and the Version of Record. Please cite this article as doi: 10.1002/uog.17498

This article is protected by copyright. All rights reserved. 
This factor is of much greater importance for evaluating histological findings in the placenta compared to those in tumour specimens, for example, since many tumours have highly characteristic features which are never seen in 'normal' patients and hence such 'abnormal' cases are easily recognised regardless of their prevalence in a population. In contrast, many of the features noted in the placentas from pregnancies complications with PET and fetal growth restriction are also reported in apparently uncomplicated pregnancies; it is the frequency and severity of such findings which are increased in PET. ${ }^{1}$ For this reason, it is apparent that if one were to take a hypothetical group of 100 placentas, in whom 90 were affected by PET (assume 50\% showed histological abnormalities) with 10 unaffected controls (assuming 10-20\% showed histological abnormalities), the 'test characteristics' for the histological feature in question to detect PET would appear good. However, if the same 90 PET cases were now included as part of a larger group of 1,000 placentas, in which the other 910 were unaffected, the success with which pathological evaluation could correctly identify PET cases would significantly reduce.

Furthermore, by definition, cases submitted for routine placental histopathological evaluation as part of clinical care are reported in full knowledge of the clinical history. This leads to significant potential for subconscious subjective bias in the recognition and reporting of features, both positive and negative depending on the clinical history. The effect of non-blinding is clearly illustrated by the findings of the present study, in which studies without pathologist blinding reported greater differences between the PET and normal outcome groups, with consequent erroneous overestimation the significance of the feature examined. ${ }^{1}$ The effect of clinical history on subjective evaluation of placentas can be further illustrated by a study in which a group of placental pathologists were provided with histological sections each accompanied by a clinical history, and asked to estimate the gestational age. Subsequent sets were circulated for similar evaluation, but unknown to those participating, these were the same as the first set but with changed clinical histories, and the clinical history provided influenced the 'histologically evaluated' gestational age. ${ }^{6}$

These factors highlight the potential methodological difficulties from unblinded retrospective histological reviews, or worse still, reviews of clinical histology reports from a diagnostic laboratory service, for research purposes. Prospective, blinded studies from unselected populations with evaluation independent of any clinical features have demonstrated that many histological features are present in both normal and complicated pregnancies, but with differing frequencies. Similar odds ratios to retrospective studies for associations with outcome and specific histological 'abnormalities' are reported but such unselected, blinded studies also provide data to determine other test characteristics. For example, in one study of $>1,000$ unselected low risk pregnancies the odds ratio for the association of villitis of unknown aetiology (VUE) and pregnancy induced hypertension was around 3, similar to case-control studies, but since VUE was also reported in clinically uncomplicated pregnancies, (which are not normally examined by diagnostic pathology departments), for any given unselected case in which VUE was present, there was a $>90 \%$ chance that this apparent abnormality was an incidental and clinically insignificant finding in an uncomplicated pregnancy rather than a marker of patients with $\mathrm{PIH}^{5}$

This article is protected by copyright. All rights reserved. 
The recognition and publication of such factors is therefore important to ensure appropriate and accurate interpretation of histological features for future studies. As illustrated by the present study, histopathological examination of the placenta can provide important information regarding underlying pathophysiological mechanisms, and in future, may guide personalised care in subsequent pregnancies. Specifically, in PET it is recognised that early onset disease is associated with more typical maternalvascular malperfusion type changes whereas late onset disease may show minimal histological abnormalities, supporting the hypothesis of differing maternal and fetal mechanisms for subtypes of PET. ${ }^{7,8}$ Nevertheless, interpretation of histological findings in individual cases remains uncertain and awareness of the potential methodological pitfalls, as highlighted by the current review, should lead to improved quality of future studies on which to base strategic decisions regarding research and patient management.

This article is protected by copyright. All rights reserved. 


\section{References}

1. Falco ML, Sivanathan J, Laoreti A, Thilaganathan B and Khalil A. Placental histopathology associated with preeclampsia: a systematic review and meta-analysis. UOG (2017).

2. Kahneman D. Thinking, fast and slow. (Penguin Books, 2012).

3. The Sveriges Riksbank Prize in Economic Sciences in Memory of Alfred Nobel 2002. Available at: http://www.nobelprize.org/nobel_prizes/economicsciences/laureates/2002/. (Accessed: 7th April 2017)

4. Dobelli, R. The art of thinking clearly. (2014).

5. Pathak S, Lees, CC, Hackett G, Jessop F \& Sebire NJ. Frequency and clinical significance of placental histological lesions in an unselected population at or near term. Virchows Arch. 459, 565-72 (2011).

6. Khong TY, Staples A, Bendon RW, Chambers HM, Gould SJ, Knowles S, Shen-Schwarz S. Observer reliability in assessing placental maturity by histology. J. Clin. Pathol. 48, 420-3 (1995).

7. Mifsud W \& Sebire NJ. Placental Pathology in Early-Onset and Late-Onset Fetal Growth Restriction. Fetal Diagn. Ther. 36, 117-128 (2014).

8. Raymond D \& Peterson EA. critical review of early-onset and late-onset preeclampsia. Obstet. Gynecol. Surv. 66, 497-506 (2011).

This article is protected by copyright. All rights reserved. 


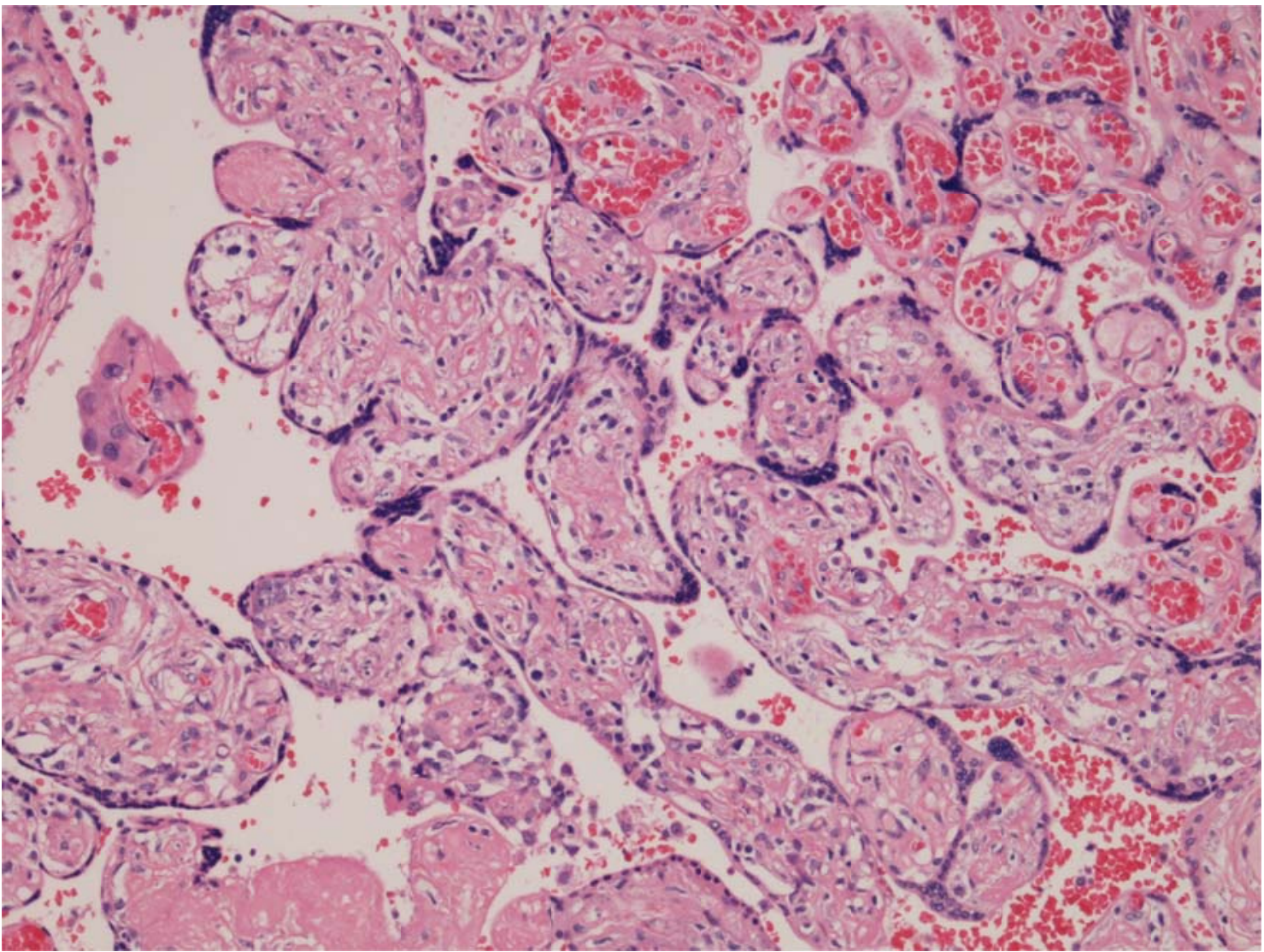

Figure 1 Photomicrograph of a term placenta with florid patchy villitis of unknown aetiology (VUE), which appears to be an entirely incidental finding; in this case the pregnancy was uncomplicated, with a normal spontaneous vaginal delivery of a normally grown infant, the placenta was submitted for examination purely on the basis of the patient having had a previous hydatidiform mole.

This article is protected by copyright. All rights reserved. 